\section{Le cil primaire, orchestrateur de la morphogenèse cérébrale}

> Dès le début des années 1960, des cils primaires, dits vestigiaux, ont été décrits dans le cerveau des mammifères. Plus récemment, des atteintes cérébrales ont été mises en évidence dans nombre de ciliopathies. Aujourd'hui, la fonction des cils primaires dans le cerveau adulte reste encore un mystère; en revanche, leur rôle au cours du développement embryonnaire a été caractérisé et s'est révélé essentiel. Depuis la neurulation et jusqu'à la naissance, quasiment toutes les cellules du cerveau en développement arborent un cil primaire, les progéniteurs en phase de prolifération comme les interneurones en cours de migration, ou encore les neurones et cellules gliales différenciés. Ce petit organite à l'interface entre la cellule et son environnement semble être impliqué dans les différentes étapes de la morphogenèse cérébrale, en particulier via son rôle dans la voie de signalisation Hedgehog (Hh).<

\section{Atteintes cérébrales des ciliopathies et modèles d'étude}

Le cerveau des mammifères est un organe hautement sophistiqué dont la morphologie reflète la diversité régionale des réseaux cellulaires. Cette fascinante complexité a été à l'origine de très nombreuses études visant à mieux comprendre les différentes étapes de la construction cérébrale. Cependant, ce n'est que récemment que le rôle du cil primaire a été mis à jour. Certes, ces petites extensions cellulaires structurées par un axonème ( 9 doublets de microtubules) et un corps basal (centriole père du centrosome) $[65,66](\rightarrow)$ avaient été décrites dans le cerveau des vertébrés dans

$(\rightarrow)$ Voir les Synthèses de C. Fort et P. Bastin ; de A. Benmerah, pages 955 et 962 de ce numéro

Cet article fait partie du numéro thématique de médecine/sciences intitulé «Cils primaires et ciliopathies $\gg\left(\mathrm{m} / \mathrm{s} \mathrm{n}^{\circ} 11\right.$, vol. 30 , novembre 2014$)$.

\section{Christine Laclef}

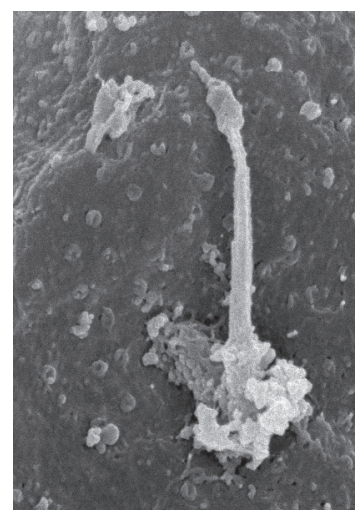

Laboratoire de biologie du développement, UPMC Université Paris 6, UMR 7622 CNRS, U969 Inserm, 9, quai Saint Bernard, 75005 Paris, France. christine.laclef@snv.jussieu.fr

les années 1960 grâce à l'avènement de la microscopie électronique à transmission. Mais, à l'époque, les cils étaient considérés comme des organites vestigiaux sans fonction particulière [1-3]. Ce n'est qu'avec l'analyse des lignées de souris dépourvues de cil et la description des syndromes humains dus à des dysfonctionnements ciliaires, que les fonctions de ce petit organite solitaire se sont révélées essentielles à de nombreux processus développementaux et, notamment, à la morphogenèse cérébrale $[4,5]$.

Les ciliopathies sont des maladies génétiques dues à des dysfonctionnements du cil d'origine variable $[6,67](\rightarrow)$.

Sur la base de phénotypes communs, on y associe des syndromes résultant d'anomalies des voies de signalisation en aval du cil [7]. De nombreuses $(\rightarrow)$ Voir la Synthèse de R. BachmannGagescu, page 1011 de ce numéro anomalies du système nerveux central (SNC) et de nombreuses manifestations cliniques neurologiques ont été décrites dans les ciliopathies (Tableau I) [8]. Cette variabilité s'explique par la nature de la mutation et/ou les atteintes combinées de plusieurs gènes. Certaines caractéristiques neuroradiologiques font partie des critères diagnostiques, comme le signe de «la dent molaire » (molar tooth sign, voir Tableau I) chez les patients atteints du syndrome de Joubert ou apparenté (JSRD, Joubert syndrome related disorders) [67] $(\rightarrow)$.

$\rightarrow$ Voir la synthèse de R. BachmannGagescu, page 1011 de ce numéro

Certaines atteintes peuvent être fréquentes, sans être constantes; c'est le cas des agénésies du corps calleux dans les syndromes OFD (oral-facial-digital), acrocalleux et hydroléthalus ou, plus rarement, dans le syndrome de Joubert. Très fréquemment, des troubles neurologiques, cognitifs ou comportementaux ont été décrits, sans pour autant que les bases neuro-anatomiques aient été systématiquement analysées. Dans tous les cas, une origine neuro-développementale de ces atteintes est possible, mais reste à déterminer. 


\begin{tabular}{|c|c|c|c|}
\hline Anomalie cérébrale & Définition & Conséquences & Ciliopathies \\
\hline Anencéphalie & $\begin{array}{l}\text { Défaut de fermeture du tube } \\
\text { neural céphalique }\end{array}$ & Absence de cerveau, décès & MKS \\
\hline Microcéphalie & Réduction du volume cérébral & $\begin{array}{l}\text { Souvent associée à un retard de } \\
\text { développement }\end{array}$ & OFD, BBS, JS, CED, LCA \\
\hline Holoprosencéphalie & $\begin{array}{l}\text { Défauts de septation médiane } \\
\text { du télencéphale, plus ou moins } \\
\text { prononcés }\end{array}$ & $\begin{array}{l}\text { Variable selon l'étendue de la } \\
\text { malformation : retard mental à décès }\end{array}$ & $\begin{array}{l}\text { MKS } \\
\text { OFD }\end{array}$ \\
\hline $\begin{array}{l}\text { Ventriculomégalie/ } \\
\text { hydrocéphalie }\end{array}$ & $\begin{array}{l}\text { Dilatation des ventricules } \\
\pm \text { augmentation de la pression } \\
\text { intracrânienne }\end{array}$ & $\begin{array}{l}\text { Dépend de l'augmentation de pression } \\
\text { intraventriculaire }\end{array}$ & $\begin{array}{l}\text { HLS } \\
\text { OFD, BBS, JS } \\
\text { JATD }\end{array}$ \\
\hline Agénésie du corps calleux & $\begin{array}{l}\text { Défaut de formation, partiel } \\
\text { ou total, de la principale } \\
\text { commissure interhémisphérique } \\
\text { du télencéphale }\end{array}$ & $\begin{array}{l}\text { Variable : asymptomatique à retards } \\
\text { mentaux sévères }\end{array}$ & $\begin{array}{l}\text { OFD, ACLS } \\
\text { JS, MKS } \\
\text { CED }\end{array}$ \\
\hline Hétérotopies & Amas de neurones ectopiques & $\begin{array}{l}\text { Souvent associées à des retards mentaux, } \\
\text { parfois cause d'épilepsie }\end{array}$ & $J S, O F D$ \\
\hline Polymicrogyrie & $\begin{array}{l}\text { Lamination corticale anormale } \\
\text { caractérisée par un excès de } \\
\text { gyration du cortex }\end{array}$ & $\begin{array}{l}\text { Variable selon l'étendue de la } \\
\text { malformation : retards mentaux, épilepsie, } \\
\text { paralysie de la face, troubles du langage, } \\
\text { etc. }\end{array}$ & MKS, JS \\
\hline Encéphalocèle & $\begin{array}{l}\text { Protrusion du cerveau et des } \\
\text { méninges hors de la boîte } \\
\text { crânienne }\end{array}$ & $\begin{array}{l}\text { Souvent accompagné d'autres } \\
\text { malformations cérébrales et cause de } \\
\text { défauts neurologiques graves }\end{array}$ & $\begin{array}{l}\text { MKS } \\
\text { JS }\end{array}$ \\
\hline Dysgénésie de l'hippocampe & Anomalie du lobe limbique & $\begin{array}{l}\text { Peut influencer l'apprentissage et les } \\
\text { fonctions cognitives }\end{array}$ & BBS, JS \\
\hline $\begin{array}{l}\text { Signe de la dent molaire: } \\
\text { hypoplasie du vermis } \\
\text { cérébelleux }\end{array}$ & $\begin{array}{l}\text { Absence partielle ou totale de } \\
\text { la région centrale du cervelet } \\
\text { (le signe de la dent molaire est } \\
\text { vu en IRM) }\end{array}$ & $\begin{array}{l}\text { Ataxies, apraxie oculomotrice et autres } \\
\text { défauts de coordination des mouvements } \\
\text { volontaires; parfois autisme et dyslexie }\end{array}$ & $\begin{array}{l}\text { JSRD } \\
\text { OFD, MKS } \\
\text { BBS, LCA }\end{array}$ \\
\hline $\begin{array}{l}\text { Malformation de Dandy } \\
\text { Walker }\end{array}$ & $\begin{array}{l}\text { Association d'un kyste de } \\
\text { la fosse postérieure du IVe } \\
\text { ventricule et d'une hypoplasie } \\
\text { du vermis cérébelleux }\end{array}$ & $\begin{array}{l}\text { Augmentation de la pression } \\
\text { intracrânienne ; retard de développement } \\
\text { moteur et de coordination des } \\
\text { mouvements, ataxies }\end{array}$ & $\begin{array}{l}\text { JS, MKS } \\
\text { JATD, PKD, NPHP }\end{array}$ \\
\hline Défauts de décussations & $\begin{array}{l}\text { Défaut de croisement de la } \\
\text { ligne médiane par certains } \\
\text { faisceaux d'axones }\end{array}$ & Mouvements en miroir & $\begin{array}{l}\text { JS (pédoncule cérébelleux } \\
\text { supérieur) } \\
\text { JS (faisceaux } \\
\text { corticospinaux) }\end{array}$ \\
\hline
\end{tabular}

Tableau I. Principales anomalies cérébrales associées aux ciliopathies. Lorsque l'anomalie cérébrale est essentielle au diagnostic, la ciliopathie est indiquée en bleu foncé gras ; lorsque l'association est fréquente, mais pas systématique, la ciliopathie est indiquée en bleu roi. Les associations occasionnelles sont indiquées en vert. Cette diversité de symptômes laisse supposer que les cils expriment différentes fonctions au cours de la construction cérébrale. (Adapté de [8].) ACLS : acrocallosal syndrome; BBS : Bardet-Biedl syndrome ; CED : cranio-ectodermic dysplasia (Sensenbrenner syndrome); HLS : hydrolethalus ; JATD : Jeune asphyxiating thoracic dystrophy ; JS : Joubert syndrome ; JSRD : Joubert syndrome related disorders ; MKS : Meckel syndrome; OFD : oro-facial-digital syndrome; PKD : polycystic kidney disease.

Comprendre comment se forme le cerveau au cours du développement embryonnaire est indispensable à la compréhension de son fonctionnement et, le cas échéant, de ses dysfonction- nements. Parmi les nombreuses approches possibles, l'analyse des phénotypes mutants, chez des modèles murins en particulier, est celle qui 


\section{Stade plaque neurale (દ8.0-ع9.0)}
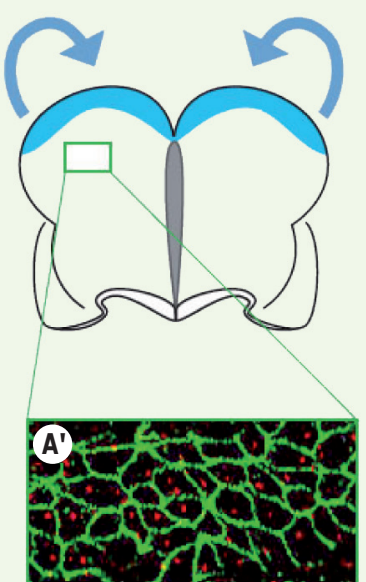

ARL13B (protéine ciliaire) ZOI (jonctions serrées)
B Stade cinq vésicules

(દ10.5-ع12.5)

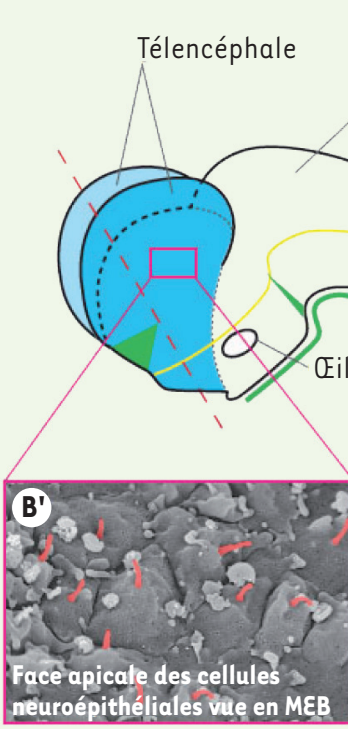

Dérivés adultes

Cortex,

Hippocampe,

Thalamus,

Hypothalamus
C

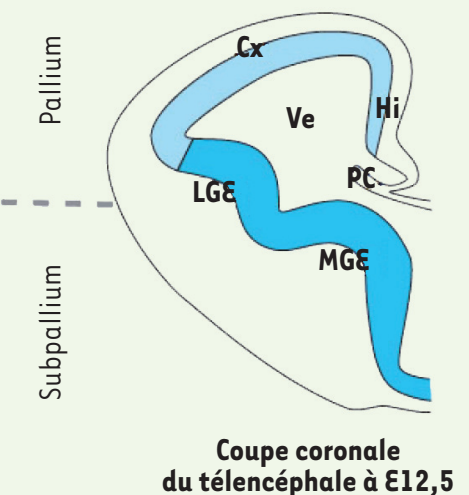

D

Mutant ciliaire :

Rpgripll Ftm/ftm $^{-}$

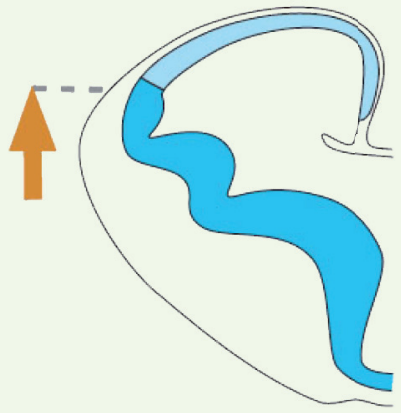

Ventralisation $\varepsilon \quad$ Mutant ciliaire : Rpgrip $1 l^{\mathrm{Ftm} / \mathrm{ftm}}$ + Gli3 $^{\Delta 699}$

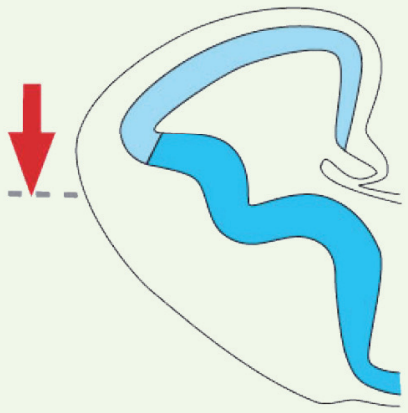

Correction

Figure 1. Rôle du cil dans la formation et la régionalisation du tube neural. $A$. Dès le stade plaque neurale ( $\varepsilon 8,0$ chez la souris), les cellules neuroépithéliales arborent un cil primaire sur leur face apicale, révélé en $A^{\prime}$ par l'immunodétection d'ARL13B, un marqueur ciliaire (en rouge; le marquage vert révèle les contours cellulaires). La zone bleue correspond à la région du télencéphale. $B$. Après le soulèvement, l'enroulement et la fusion des bourrelets neuraux, le cerveau embryonnaire se subdivise en cinq vésicules, qui prédéfinissent les grandes régions du cerveau adulte (indiquées en bleu à droite). Les structures produisant la molécule de signalisation Shh sont indiquées en vert. Les cellules neuroépithéliales possèdent toujours un cil apical, identifiable en $B^{\prime}$ en microscopie électronique à balayage (MEB). C. Les différentes régions du télencéphale en développement sont indiquées sur une demie coupe coronale; dans la région dorsale : le cortex (Cx), l'hippocampe (Hi) et le plexus choroïde (PC) ; dans la région ventrale : les éminences ganglionnaires latérale ( $L G E)$ et médiane (MGE); Ve : ventricule ; en bleu clair : le pallium ; en bleu vif : le subpallium. D. Les mutants ciliaires Rpgrip $1 l^{\text {Ftm }}$ présentent une ventralisation du télencéphale [20], un phénotype également présent chez d'autres mutants présentant des dysfonctionnements ciliaires d'origines différentes, ainsi que chez les mutants Gli3. $\varepsilon$. La ventralisation des mutants ciliaires Rpgrip Il $l^{F t m}$ est corrigée par l'apport de protéine Gli3-R [20] (par croisement avec la lignée Gli3 ${ }^{\Delta 699}$ qui exprime une forme tronquée de Gli3 fonctionnellement semblable à Gli3-R : knock-in Gli3 ${ }^{\Delta 699}$ au locus Gli3 [23]). 
a le plus apporté à l'étude de la morphogenèse cérébrale [9]. Rappelons que les mutants ciliaires, par définition, présentent une altération de la structure et/ou de la fonction du cil, qui se manifeste chez la souris par une association de phénotypes récurrents: défauts de latéralité et défauts de régionalisation (patterning) des bourgeons de membres et du tube neural spinal [10]. Cette triade constitue la signature phénotypique des mutants ciliaires, et les autres phénotypes, en particuliers cérébraux, ont parfois été occultés. Surtout, nombre de ces mutants ciliaires meurent en milieu de gestation (entre $\varepsilon 10$ et $\varepsilon 13$ chez la souris). Dans ce cas, l'analyse des étapes ultérieures du développement ne peut se faire qu'en analysant des mutants hypomorphes ou des animaux chez lesquels un knock-out (KO) conditionnel du gène est réalisé. Cette revue fait le point sur les données, les hypothèses et les questions relatives au rôle du cil primaire dans la formation du cerveau et, plus particulièrement, du cerveau antérieur.

\section{Rôle du cil dans la formation et la régionalisation du cerveau antérieur}

Les milliards de neurones et de cellules gliales qui constituent le cerveau des mammifères sont générés à partir du feuillet ectodermique de l'embryon. La plaque neurale, induite lors de la gastrulation, contient les cellules neuro-épithéliales, dont les changements de forme et d'adhésivité vont contribuer à la formation du tube neural. Dès le début de la neurulation ( $\varepsilon 8,0$ chez la souris), ces cellules arborent un cil primaire sur leur face apicale (Figure 1A). Ces cils baignent initialement dans la cavité amniotique, puis feront face ensuite à la cavité centrale du tube neural après le soulèvement et la fusion des bourrelets neuraux le long de la ligne médiane.

\section{Cil primaire et fermeture du tube neural}

Si la fermeture du tube neural ne s'opère pas correctement, le développement ultérieur du système nerveux central s'en trouve compromis. Or, plusieurs mutants ciliaires présentent un phénotype attestant d'un défaut de fermeture du tube neural antérieur (exencéphalie), qui s'accompagne d'un développement extériorisé du cerveau chez la souris: Dynchc2/Dync2hl (dynein, cytoplasmic 2, heavy chain 1), Ift52 (intraflagellar transport protein 52), Ift57 hippi, Ift88 flexo , Ift122, Ift172 ${ }^{\text {wimple }}$, Mks I (Meckel syndrome, type 1), Inpp5e (inositol polyphosphate-5-phosphatase), Ftm/Rpgrip Il (retinitis pigmentosa GTPase regulator interacting protein 1l), Tmem67 (transmembrane protein 67), Tulp3 (Tubby-like protein 3), etc. [11]. Chez l'homme, des défauts équivalents conduisent à des anencéphalies, un signe clinique rare, décrit parfois chez des fœtus atteints du syndrome de Meckel ou du syndrome acrocalleux [2]. Ainsi, le cil primaire semble impliqué dans la fermeture du tube neural antérieur de la souris, mais par quels mécanismes? Cela reste à établir précisément [12]. II est à noter également que ces mutations ne sont pas totalement pénétrantes. L'incidence de l'exencéphalie est, par exemple, de $77 \%$ chez les souris
Tulp3 $3^{-/-}, 37 \%$ pour la mutation $\mathrm{Ftm}^{-/-}, 30 \%$ pour la mutation Inpp $5 e^{-/-}$, ou encore $28 \%$ chez les animaux $\mathrm{Mksl}^{-/-}$[11]. De plus, le fond génétique influence considérablement la fréquence de ce phénotype, ce qui pourrait indiquer un déterminisme multifactoriel et/ou des sensibilités différentes aux subtiles variations stochastiques qui surviennent lors du développement.

Dès sa formation, le tube neural présente des différences morphologiques le long des axes antéro-postérieur (AP) et dorso-ventral (DV). La partie antérieure du tube neural se caractérise par des renflements clairement visibles, qui correspondent aux futures subdivisions morphologiques du cerveau (Figure lB). La partie postérieure, plus fine, est à l'origine de la moelle épinière. Les processus de régionalisation sont conditionnés par des signaux morphogènes et l'expression de gènes régulateurs (facteurs de transcription) qui dictent le destin et le comportement ultérieur des cellules neurales.

\section{Le cil primaire, un acteur clé de la signalisation Hedgehog}

Dans le tube neural postérieur, la spécification des types cellulaires ventraux est dépendante de la production de sonic hedgehog (Shh, principale protéine de la famille Hh produite dans le SNC). Shh est sécrétée par les cellules ventrales de la plaque du plancher et constitue un gradient morphogène ventralisant [13]. Or, chez un grand nombre de mutants ciliaires, une réduction, voire une absence, de spécification de certains types cellulaires ventraux ont été décrites [68] $(\rightarrow)$.

$(\rightarrow)$ Voir la Synthèse de C. Métin, page 991 de ce numéro

Cette dorsalisation du tube neural spinal atteste d'un défaut d'activation de la voie de signalisation Hh dans les territoires ventraux [14]. C'est dans ce contexte développemental que le cil primaire s'est révélé être un élément essentiel dans la perception et la transduction du signal Hh chez les mammifères $[14,15]$. Le cil est alors apparu comme une «antenne cellulaire » dédiée à la réception des signaux de l'environnement.

Cependant, en absence de signal $\mathrm{Hh}$, le cil primaire contrôle l'activité des effecteurs transcriptionnels de la voie Hh que sont les protéines Gli (famille de facteurs de transcription à doigt de zinc associées aux gliomes, voir Figure 2). En particulier, le clivage protéolytique de la protéine Gli3, qui conditionne son activité répresseur, est dépendant de l'intégrité ciliaire. Ainsi, une réduction de la forme tronquée de la protéine Gli3 (Gli3-R) est fréquemment observée chez les mutants ciliaires. Cette fonction du cil, indé- 


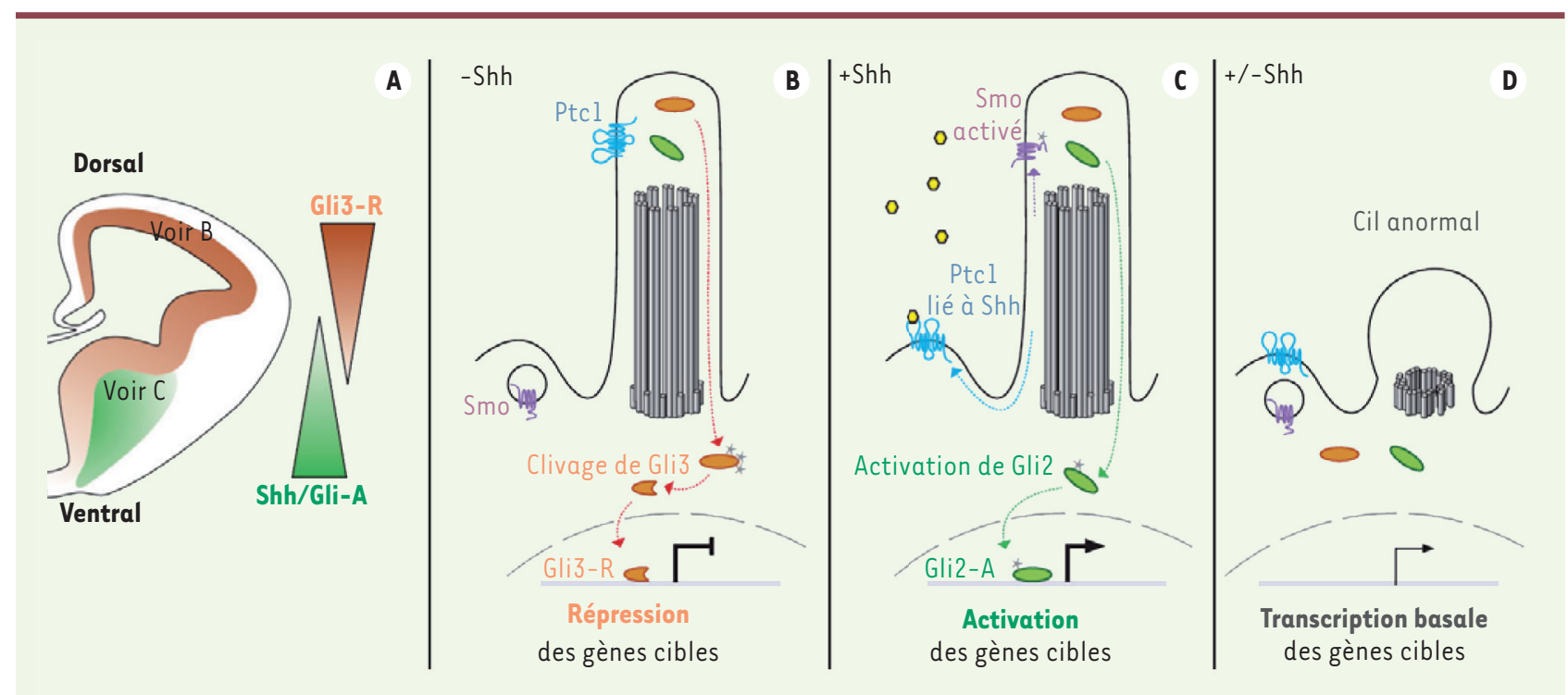

Figure 2. Le cil primaire et la transduction du signal Shh dans le télencéphale. A. La molécule de signalisation Shh et les formes activatrices des facteurs Gli (Gli-A) se trouvent principalement dans la région ventrale du télencéphale, alors que la forme inhibitrice de Gli3 (Gli3-R) est essentiellement présente dans la région dorsale du télencéphale. Chez les mammifères, il a été montré que les acteurs moléculaires de la transduction du signal Shh transitent dans le cil primaire, et que ce dernier est indispensable à l'activité transcriptionnelle des protéines Gli2 et Gli3, facteurs de transcription à doigts de zinc. B. En l'absence de ligand Shh, le récepteur membranaire Patchedl (Ptcl) est présent à l'apex du cil, ainsi que les protéines Gli2 (vert) et Gli3 (orange) ; la protéine transmembranaire Smoothened (Smo) reste hors du cil. La protéine Gli3 subit des modifications post-traductionnelles $(*)$ et un clivage protéolytique qui potentialise son activité de répresseur transcriptionnel (Gli3-R). Cette maturation de Gli3 est dépendante du cil primaire. C. En présence de ligand Shh, le complexe Ptcl-Shh quitte le cil, alors que Smo s’y accumule. Le clivage protéolytique de Gli3 est inhibé. Les protéines Gli subissent une modification post-traductionnelle qui potentialise leur fonction activatrice de la transcription (Gli-A). Cette maturation des Gli est dépendante du cil primaire. D. En l'absence de cil primaire, les fonctions activatrice et inhibitrice des protéines Gli sont compromises.

pendante de $\mathrm{Hh}$, est déterminante pour la régionalisation dorsoventrale dans la partie antérieure du tube neural et antéro-postérieure dans les bourgeons des membres. En effet, plusieurs mutants ciliaires présentent des défauts de régionalisation dorso-ventrale du télencéphale. Il s'agit de mutants déficients pour différents gènes, tous impliqués dans la formation ou la fonction du cil, codant pour des protéines apposées au corps basal (Ftm/Rpgripll, Ofdl), des IFT (Ift88 Cobblestone, Ift139alien, Ift172) ou des sous-unités de moteurs moléculaires (Dynchc2, Kif3a [kinesin family member 3A]) impliqués dans le transport intraflagellaire antérograde (Ift88, Ift172, Kif3a) ou rétrograde (Ift139, Dynchc2) [16-21, 65] $(\rightarrow)$.

$(\rightarrow)$ voir la synthèse de C. Fort et P. Bastin, page 955 de ce numéro Tous ces mutants présentent une ventralisation du télencéphale, c'est-à-dire une extension du territoire ventral (subpallium) au détriment du territoire dorsal (pallium), et la frontière entre les deux s'estompe (présence ectopique de cellules d'identité ventrale dans le territoire dorsal) (Figure 1). Ainsi, l'altération du cil peut conduire à la fois à une ventralisation du télencéphale et à une dorsalisation du tube neural postérieur. Ceci s'explique par le fait que les effecteurs principaux de la voie $\mathrm{Hh}$ ne sont pas les mêmes dans ces deux régions du SNC (rôle majeur de Gli2 dans le tube neural spinal et de Gli3 dans le télencéphale).
La ventralisation du télencéphale des mutants ciliaires ressemble à ce qui a été décrit chez les mutants Gli3 [22], anomalies qui avaient conduit à définir cet effecteur de la voie Hh comme un acteur majeur de la spécification du télencéphale dorsal [9]. Ainsi, il semblait raisonnable de postuler que les cils primaires étaient nécessaires à la spécification du télencéphale dorsal via leur rôle dans la production de Gli3-R. Nous avons formellement validé cette hypothèse en réintroduisant la protéine Gli3-R chez les mutants ciliaires Rpgrip I/ Ftm/Ftm en croisant ces souris avec la lignée Gli $3^{\Delta 699}$ qui exprime une forme tronquée de Gli3 fonctionnellement semblable à Gli3-R (knock-in Gli3 ${ }^{\Delta 699}$ au locus Gli3) [23]. L'introduction d'un allèle $G l i 3^{\Delta 699}$ chez les mutants Rpgrip $1 l^{\text {Ftm/Ftm }}$ est suffisante pour rétablir la régionalisation dorso-ventrale du télencéphale à $\varepsilon 12,5$. Cette correction phénotypique a lieu malgré la persistance des altérations ciliaires et montre qu'il est possible de restaurer la fonction des cils primaires in vivo en ciblant l'effecteur maître en aval des cils, ici Gli3-R. De plus, par la même approche, nous avons 


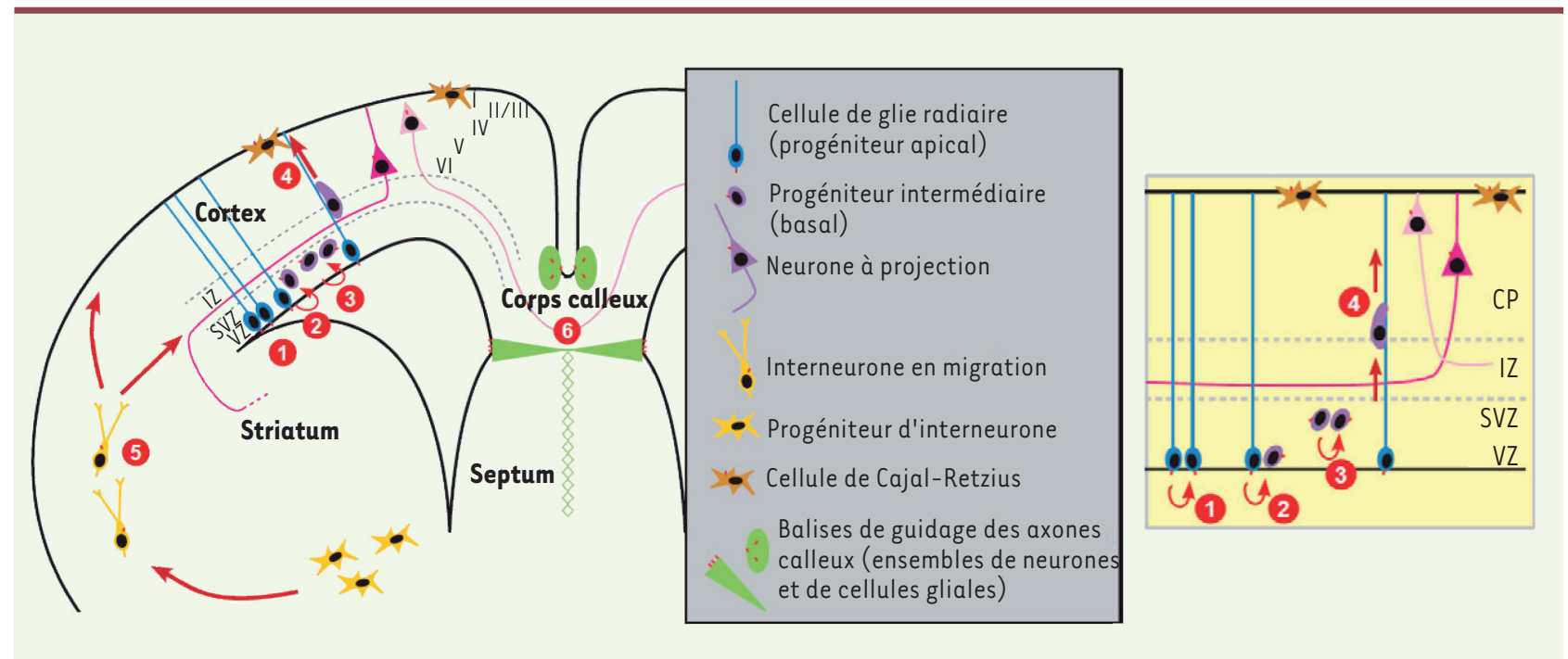

Figure 3. Participation de nombreux types cellulaires ciliés à la formation du cortex cérébral. Le cortex cérébral adulte est le support de processus mentaux sophistiqués qui reposent sur une organisation cyto-architecturale particulière. Les six couches de neurones caractéristiques du cortex adulte se mettent en place progressivement pendant la seconde moitié de la gestation chez la souris, selon un processus dit «inside-out » où les couches profondes se différencient avant les couches périphériques (VI à II/III). Le cortex contient une grande diversité de neurones qui peuvent être regroupés en deux grandes classes : les neurones à projection et les interneurones. Les neurones à projection, de forme pyramidale, possèdent de longs axones qui établissent des connexions avec des cibles éloignées; ils produisent en général des neurotransmetteurs excitateurs (type glutamate). Les neurones à projection ont une origine dorsale. En début de neurogenèse, des divisions symétriques multiplient le pool des cellules de glie radiaire (aussi appelées progéniteurs apicaux) (1) dans la zone ventriculaire (VZ). Puis, des divisions asymétriques (2) génèrent des progéniteurs intermédiaires (aussi appelés progéniteurs basaux) qui subissent un ou plusieurs cycles de divisions supplémentaires (3) dans la zone sous-ventriculaire (SVZ). Les différentes populations de neurones corticaux se différencient successivement et se positionnent dans les différentes couches de la plaque corticale par migration radiaire (4); un processus qui dépend à la fois des cellules de la glie radiaire (qui s'étendent dans l'épaisseur du cortex, et possèdent des points d'attache ventriculaire et pial) et des cellules de Cajal-Retzius (couche I, produisant la reelin en périphérie du cortex) [63]. Les interneurones, quant à eux, établissent des microcircuits locaux et produisent en général des neurotransmetteurs inhibiteurs (GABA [acide $\gamma$-aminobutyrique]). Ils ont une origine ventrale (éminence ganglionaire médiane) et parcourent un long chemin par migration tangentielle (5) pour rejoindre le cortex où ils finissent par se positionner dans les différentes couches corticales [64]. Par ailleurs, une part importante du SNC est occupée par des cellules gliales ou parcourue de faisceaux d'axones (corps calleux, striatum, zone intermédiaire, etc.). Certaines cellules gliales participent au guidage axonal ; seules celles participant au guidage des axones calleux sont indiquées sur le schéma (6).

montré que la morphogenèse des bulbes olfactifs, altérée par la ventralisation du télencéphale chez le mutant Rpgripl $1 l^{\mathrm{Ftm} / \mathrm{Ftm}}$, est restaurée par l'apport de Gli3-R (par l'allèle Gli3 ${ }^{\Delta 699}$ ) [20]. Dans un autre contexte, Gli $3^{\Delta 699}$ corrige le phénotype rénal des mutants Kif3a [24]. Cependant, Gli $3^{\Delta 699}$ ne corrige pas tous les phénotypes des mutants ciliaires et, au contraire, pourrait exacerber certains défauts (au niveau des membres par exemple).

Une autre tentative de correction est illustrée par l'étude du mutant Iftl39alien. Le phénotype de ce dernier présente la particularité, au niveau du tube neural postérieur, d'être corrélé à une activation accrue de la voie Hh (ventralisation), et non pas à une réduction comme c'est le cas chez la plupart des autres mutants [25]. Ici, le but a donc été de réduire la signalisation Hh, en croisant les souris Ift139alien avec les lignées KO pour Shh ou Gli2. Les corrections phénotypiques observées chez les doubles mutants
Ift139alien/alien $\mathrm{Shh}^{+/-}$et Ift139alien/alien $\mathrm{Gli2}^{+/-}$, respectivement dans le télencéphale et le tube neural postérieur, semblent conforter l'idée que le défaut résulte ici d'un excès de signal Hh et d'une surproduction de la forme activatrice de Gli2 [19, 25]. Des défauts ciliaires pourraient ainsi conduire, selon le type de mutation, soit à l'activation de l'activateur Gli2 (Ift139alien), soit à l'inhibition du répresseur Gli3 (Ftm/Rpgripll). Or, l'équilibre entre ces deux formes actives des protéines Gli (activateur/répresseur) est déterminant dans de nombreux processus développementaux.

D’autres défauts cérébraux précoces ont été décrits chez les mutants ciliaires, comme une holoprosencéphalie semilobaire (défaut de septation médiane du 
télencéphale) chez les mutants Ift172 et Ttbk2 (définie au stade $\varepsilon 10,5)[17,26]$. De plus, des défauts de frontière le long de l'axe antéro-postérieur ont été rapportés: entre le télencéphale et le diencéphale chez les mutants Ift88cobblestone et Ift $139^{\text {alien }}[18,19]$, et entre le mésencéphale et le métencéphale dans le knock-out Ift172 [17].

Ces défauts de régionalisation peuvent avoir des conséquences dramatiques sur les étapes suivantes de la construction du cerveau embryonnaire. En effet, les différentes régions spécifiées possèdent d'emblée des propriétés spécifiques qui conditionnent leur développement ultérieur en termes de prolifération, différenciation, migration cellulaire, navigation axonale et synaptogenèse. Le cil primaire semble intervenir également dans le contrôle de ces différents comportements cellulaires, indépendamment de son rôle dans la régionalisation.

\section{Rôle du cil dans la neurogenèse cérébrale}

Depuis la neurulation et jusqu'à la naissance, quasiment toutes les cellules du SNC en développement arborent un cil primaire : pas seulement les neurones différenciés, mais également les progéniteurs neuraux en phase de prolifération et les précurseurs d'interneurones pendant leur migration (Figure 3 ).

\section{Cil primaire et transition prolifération/différenciation des progéniteurs neuraux}

Dans les progéniteurs neuraux, le centrosome est alternativement requis pour ancrer le cil primaire (pendant l'interphase) et pour organiser les fuseaux de division (en mitose) ; le corps basal du cil n'est autre que le centriole père du centrosome. II existe donc un lien étroit entre le cil et le cycle cellulaire $[27,69](\rightarrow)$. $\rightarrow$ Voir la Synthèse de N. Delgehyr et N. Spassky, page 976 de ce numéro
Cependant, selon les modèles cellulaires considérés, les conclusions semblent diverger. Dans certains cas, la présence du cil ralentirait la progression du cycle, ce dernier s'accélérant chez les mutants ciliaires. C'est ce qui a été montré dans le cortex du mutant conditionnel nestin-Cre; Kif3a flox [21]. Dans d'autres cas, la présence du cil est nécessaire pour maintenir un nombre important de cellules en division, via la transduction du signal Shh qui a un effet mitogène dans ce contexte. C'est le cas, par exemple, des progéniteurs des grains dans le cervelet, dont le nombre est réduit chez les mutants conditionnels Ift $88^{\text {flox }}$ et Kif $3^{\text {flox }}$ (dont l'invalidation est ciblée par hGFAP [human glial fibrillary acidic protein](re) $[28,29]$. Ces défauts sont à corréler avec les hypoplasies du vermis cérébelleux (région centrale du cervelet) décrites dans plusieurs ciliopathies (BBS [Bardet-Biedl syndrome], OFD, néphronophtise, syndrome de Jeune) [30] (Tableau l).

En plus de son influence directe sur la durée des cycles cellulaires et sur le nombre de cellules en division, le cil primaire serait un élément essentiel dans l'équilibre entre divisions symétriques/ asymétriques lors de la neurogenèse corticale (Figure 3). Lors des divisions asymétriques, la cellule fille, qui hérite du centriole père le plus âgé, reforme plus rapidement un cil primaire, qui d'ailleurs ne disparaît pas forcément totalement pendant la mitose [31]. Elle pourrait ainsi répondre plus rapidement aux signaux tels que Shh [32]. De plus, le centriole père, le plus âgé, ségrège plus fréquemment dans les cellules gardant leur caractère progéniteur (plutôt que dans les cellules s'engageant dans la différenciation neuronale) [33]. En conclusion, les cils primaires, par leur interaction avec le centriole père du centrosome, sont liés à la transition prolifération/différenciation des progéniteurs neuraux qui influencent directement la neurogenèse corticale.

Le cil primaire, producteur de signaux?

Dès le début de la neurogenèse, les progéniteurs neuraux, aussi appelés cellules neuroépithéliales, forment un épithélium pseudostratifié homogène: toutes les cellules présentent les mêmes caractéristiques de polarité apicobasale, le cil se trouvant sur la face apicale. Les futurs neurones corticaux se détachent ensuite de la zone apicale pour migrer et se différencier en périphérie. Un des tout premiers signes distinctifs de l'engagement des cellules vers un destin neuronal serait la formation d'un cil en position basolatérale, et non plus en position apicale [34]. Récemment, une étude réalisée au niveau du tube neural postérieur a montré l'existence d'un mécanisme d'abscission apicale, jusque là inconnu [35]. La partie apicale de la cellule, comprenant le cil et les jonctions serrées, reste à sa place, alors qu'un clivage s'opère au niveau des jonctions adhérentes (constriction actine/myosine/N-cadhérine/ $\alpha P K C$ [protéine kinase $[\alpha]$ ) pour libérer la cellule de son ancrage apical. Lors de cette scission cellulaire, les centrioles se déplacent vers le corps cellulaire, alors que les marqueurs ciliaires analysés restent dans la particule apicale. Finalement, cette perte rapide de polarité apicale permet au futur neurone de se déplacer dans l'épaisseur du neuroépithélium, d'acquérir de nouvelles caractéristiques morphologiques et fonctionnelles, bref de se différencier, et cela selon toute vraisemblance en l'absence de cil primaire, au moins dans un premier temps. Le devenir de la particule apicale n'a pas été exploré, mais elle pourrait être libérée dans la lumière du tube neural qui contient le liquide cérébrospinal (LCS), ce dernier véhiculant, de fait, toutes sortes de composants moléculaires et vésiculaires [36]. Parmi ces composants, certains pourraient être produits par le cil lui-même, comme semblent l'indiquer les figures de bourgeonnement observées à l'extrémité du cil [37]. 
Si cette fonction de sécrétion se confirmait, cela impliquerait que les cils ne sont pas seulement des antennes réceptrices, mais qu'ils pourraient également participer à la production de signaux.

\section{Protéine ciliaire ARL13B et polarité apico-basale}

Par ailleurs, l'établissement de la polarité apicobasale des cellules neuroépithéliales au début de la neurogenèse corticale dépend de la protéine ciliaire Arl13b (ADP-ribosylation factor-like 13B) (GTPase de la famille ARF/ARL) [38]. Chez le mutant nul Arll3b hennin, la polarité se trouve complètement inversée: le pôle apical se retrouve en périphérie et le pôle basal fait face aux cavités ventriculaires. Conformément à cette inversion, les progéniteurs sont en périphérie et les neurones corticaux migrent et se différencient successivement dans des couches plus internes, en parfaite opposition avec ce qui se passe en situation normale. L'utilisation de knock-out conditionnels a permis de montrer que Arl13b est critique pour l'acquisition de la polarité apico-basale entre $\varepsilon 9,0$ et $\varepsilon 10,5$. Néanmoins, il n'est pas certain que ce phénotype soit véritablement dû à un dysfonctionnement du cil. À ce jour, ce phénotype radical n'a pas été retrouvé chez d'autres mutants ciliaires (Ift88 Cobblestone, RpgripIl/Ftm, Kif3a, Rfx [regulatory factor 3X], etc.). En effet, l'identification d'un phénotype comme « ciliaire » réside dans son caractère commun, partagé avec d'autres mutants dont les anomalies ciliaires résultent de mutations différentes [65].

Les différences phénotypiques, en revanche, peuvent être dues à des fonctions spécifiques de certaines protéines ciliaires, ou bien à des fonctions extraciliaires de la protéine mutée $[70](\rightarrow)$.

Or, nombre de fonctions non ciliaires (KIF3A, IFT88, etc.) ont été attribuées à des protéines ciliaires [39, 40].

En conclusion, la position du cil primaire semble refléter les propriétés de polarité apicobasale des cellules neurales et varie lors de leur différenciation. De plus, le cil, du fait de son lien intime avec le centrosome, semble également important dans l'organisation et la dynamique du cytosquelette, essentielles notamment aux migrations cellulaires des futurs neurones.

\section{Cils, migrations neuronales et projections axonales}

Pendant longtemps, on a pensé que les cellules en migration étaient dépourvues de cil. On pensait que le centrosome ne pouvait pas s'ancrer à la membrane pour soutenir un cil alors qu'il était engagé dans l'organisation et le remaniement dynamique des microtubules conduisant à la progression des protrusions cellulaires et à la translocation nucléaire. Et pourtant, deux publications ont montré la présence et l'importance du cil primaire des interneurones pendant leur migration tangentielle vers le cortex $[41,42,68](\rightarrow)$.

$(\rightarrow)$ Voir la Synthèse de C. Métin, page 991 de ce numéro

L'absence ou le dysfonctionnement du cil résultant de l'invalidation conditionnelle des gènes Kif3a, Ift88 ou Arl13b perturbent la migration des interneurones, en causant un allongement des périodes de pause, une augmentation du nombre de neurites et une stabilisation de ces protrusions. De plus, l'orientation de la migration est altérée dans le cortex, ce qui conduit à un positionnement anormal des interneurones dans les couches corticales. La voie de signalisation Shh semble impliquée dans ces défauts de migration, mais elle n'est probablement pas la seule en cause.

Enfin, dès leur positionnement dans les différentes couches du cortex, les neurones pyramidaux vont produire des axones dont la croissance orientée permet l'établissement de connexions synaptiques avec d'autres neurones corticaux (projections cortico-corticales) ou avec des cibles se trouvant dans des territoires postérieurs du SNC (projections corticofuges) [43]. La grande majorité des projections cortico-corticales interhémisphériques transitent par le corps calleux (Figure 3) [44]. Or, des agénésies du corps calleux sont fréquemment observées dans certaines ciliopathies (OFD, syndrome de Jeune, syndrome de Meckel, syndrome acrocalleux) (Tableau I) [8]. L'analyse de deux mutants ciliaires, $R f x 3$ et Rpgripll/Ftm, a révélé une agénésie du corps calleux résultant de défauts de positionnement des cellules de guidage qui jalonnent la trajectoire empruntée par les axones calleux. Ces cellules se différencient en position ectopique chez les mutants ciliaires, probablement suite au défaut de régionalisation précoce du télencéphale. Ce phénotype, semblable à celui qui est décrit chez les mutants Gli3 [22], peut être corrigé par la réintroduction de Gli3-R chez les doubles mutants $\mathrm{Ftm}^{-/-} \mathrm{Gli}^{3^{4699}}$ et $\mathrm{Rf} \times \mathrm{3}^{-/-}$ Gli3 $3^{\Delta 699}$ [45] (C. Laclef, manuscrit en préparation).

De plus, chez ces mutants, existent également des défauts des projections corticofuges (structure très anormale du striatum), dont l'origine reste à analyser. Enfin, chez le mutant Bbs8, un défaut de fasciculation et de projection des axones issus de neurones sensoriels olfactifs a été mis en évidence [46]. Ainsi, de nombreux défauts de projections axonales sont en cours d'étude chez les modèles murins de ciliopathies, qui sont à mettre en parallèle avec les défauts de décussation (croisement de la ligne médiane), décrits notamment chez des patients atteints du syndrome de Joubert [47, $48,65]$.

\section{Cils et hydrocéphalie}

Une ventriculomégalie (dilatation des cavités ventriculaires) est associée à certaines ciliopathies (BBS, HLS [hydrolethalus syndrome]; voir Tableau I), mais son origine chez l'homme est probablement distincte de celle des hydrocéphalies identifiées chez les mutants ciliaires murins (Iftl88 ORPK , Rpgripl/ Itm, $R f \times 3, \operatorname{Inpp} 5 e$, etc.). L'hydrocéphalie est une ventriculomégalie due à une augmentation de la pression 
intraventriculaire, qui peut avoir des conséquences dramatiques sur le tissu neural adjacent. On distingue les hydrocéphalies obstructives et communicantes, selon qu'il y a, ou non, occlusion des voies de circulation du liquide céphalospinal. Parmi les multiples causes d'hydrocéphalie postnatale citons les défauts des battements ciliaires coordonnés qui mettent en mouvement le liquide céphalospinal. Mais, chez la souris, ces cils mobiles qui tapissent les ventricules ne se forment que lorsque les cellules épendymaires se différencient, c'est-à-dire dans les deux semaines qui suivent la naissance [49]. Ils ne peuvent donc pas être en cause dans les hydrocéphalies anténatales. Dans ce cas, celles-ci peuvent s'expliquer par un déséquilibre entre la production (excessive) et la résorption (insuffisante) du liquide céphalospinal. Les cellules du plexus choroïde, qui sécrètent le liquide céphalospinal, portent des touffes de cils primaires, une caractéristique atypique, car les cils primaires sont en général solitaires [50]. Ces cils primaires réguleraient à la fois la quantité de liquide céphalospinal sécrété (par un mécanisme impliquant le récepteur au neuropeptide FF) et sa composition (accumulation d'ions chlorure chez les mutants Ift88 ORPK) [50, 51].

Par ailleurs, l'hydrocéphalie des souris Bbs $1^{\text {M390R/M390R }}$, caractérisées par la mutation la plus fréquemment identifiée chez les patients BBS, peut être partiellement corrigée (50\%) par l'administration de lithium aux mères pendant la gestation [52]. Le lithium avait déjà été mentionné comme agoniste de la voie Wnt, capable de corriger des défauts de prolifération au niveau du cervelet, chez le mutant Ahil [53]. L'ion lithium est connu pour ses effets pléiotropes: il agit notamment sur la régulation de l'activité de la sérine thréonine kinase, GSK3 $\beta$ (glycogen synthase kinase $3 \beta$ ), impliquée, entre autres, dans les voies de signalisation PDGF $\alpha$ (plateled-derived growth factor $\alpha$ ), Wnt/ $\beta$-caténine et dans le clivage de Gli3 (voie $H h$ ). Ces travaux ouvrent donc des perspectives thérapeutiques intéressantes, mais la compréhension des mécanismes sous-jacents nécessitera des études plus approfondies.

\section{Perspectives pour les fonctions postnatales du cil dans le cerveau}

Le cil primaire intervient clairement dans différentes étapes du développement cérébral anténatal, mais également dans le cerveau postnatal. La maturation des neurones se poursuit après la naissance, notamment avec l'établissement des connexions synaptiques. Or, il semble exister une corrélation entre la richesse des arborisations dendritiques et le cil primaire [54, 55]. Certaines régions cérébrales contiennent des cellules souches neurales dotées d'un cil qui génèrent de nouveaux neurones pendant toute la vie adulte. Or, chez les mutants Kif3a et Stumpy, la neurogenèse dans I'hippocampe est réduite, conséquence d'un défaut de signalisation $\mathrm{Hh}[56,57]$. Les cils primaires ont été décrits sur différents types cellulaires dans quasiment toutes les régions du cerveau adulte et ils sont souvent enrichis en récepteurs membranaires couplés aux protéines $G[58,59]$. Indirectement, l'étude des mutants ciliaires obèses a permis de définir une fonction du cil dans le contrôle hypothalamique de la satiété $[60,61$, 71] $(\rightarrow)$.

Mais, de manière générale, le

rôle joué par ce petit organite dans un océan de dendrites et d'axones reste encore bien mystérieux. Ce petit compartiment isolé pourrait-il concentrer et intégrer des signaux annexes, extrasynaptiques, qui contribuent à la survie, à la plasticité, voire à la fonction des neurones? Plusieurs maladies neurodégénératives ont été associées à des dysfonctionnements ciliaires. La dégénérescence des photorécepteurs de la rétine est très fréquente dans les ciliopathies. Le cil pourrait aussi être en cause dans les ataxies spinocérébelleuses $[26,62]$, la chorée de Huntington, la maladie de Parkinson, ou encore certains syndromes neurologiques tels que la schizophrénie ou la dyslexie $[2,5]$. Les recherches futures, notamment I'exploitation d'animaux chez lesquels un knock-out conditionnel permettrait de cibler l'invalidation des gènes ciliaires dans le cerveau adulte, devraient offrir un nouvel éclairage sur ces questions. $\diamond$

\section{SUMMARY}

Primary cilia control different steps of brain development

The role of primary cilia in adult neurons remains elusive, however their developmental functions during brain morphogenesis have been recently highlighted thanks to mouse models. Unmistakably, they are needed for Hedgehog ( $\mathrm{Hh}$ )-dependent patterning in the forebrain. Not only for $\mathrm{Hh}$ reception itself, but most importantly for a downstream event in the Hh transduction pathway, independent of Hh ligand: the Gli3 processing. Indeed, phenotypes due to cilia disruption in the developing brain, such as early patterning, olfactory bulb or corpus callosum formation, can be rescued by reintroducing Gli3-R (the short truncated form of Gli3 working as a transcriptional repressor of Hh target gene). In addition, primary cilia control the proliferation rate in different neural progenitors in the cortex, the hippocampus and the cerebellum; they are required for proper migration of interneurons. And cilia dysfunction is correlated with hydrocephaly, synaptogenesis defects and aberrant axonal tract projections. Most of these neurodevelopmental defects can be related to the various neurological features frequently observed across the ciliopathy spectrum. And thus, understanding the underlying mechanisms of these diverse functions of primary cilia in the brain is a new fundamental challenge. $\diamond$ 


\section{LIENS D'INTÉRÊT}

L'auteur déclare n'avoir aucun lien d'intérêt concernant les données publiées dans cet article.

\section{RÉFÉRENCES}

1. Fuchs JL, Schwark HD. Neuronal primary cilia: a review. Cell Biol Int $2004 ; 28: 111-8$.

2. Louvi A, Grove EA. Cilia in the CNS: The quiet organelle claims center stage. Neuron 2011 ; 69 : 1046-60.

3. Bloodgood RA. From central to rudimentary to primary: the history of an underappreciated organelle whose time has come. The primary cilium. Methods Cell Biol $2009 ; 94: 2-52$.

4. Han YG, Alvarez-Buylla A. Role of primary cilia in brain development and cancer. Curr Opin Neurobiol $2010 ; 20: 58-67$.

5. Willaredt MA, Tasouri $\varepsilon$, Tucker KL. Primary cilia and forebrain development. Mech Dev 2013 ; $130: 373-80$.

6. Badano JL, Mitsuma N, Beales PL, Katsanis N. The ciliopathies: an emerging class of human genetic disorders. Annu Rev Genom Human Genet 2006 ; 7 : 125-48.

7. Baker K, Beales PL. Making sense of cilia in disease: the human ciliopathies. Am J Med Genet $C$ Semin Med Genet 2009 ; 151C : 281-95.

8. Baker K, Beales PL. Abnormalities of the central nervous system across the ciliopathy spectrum. In : Tucker KL, Caspary T, eds. Cilia and nervous system development and function. New York : Springer, $2013: 229-73$.

9. Hébert JM, Fishell G. The genetics of early telencephalon patterning: some assembly required. Nat Rev Neurosci $2008 ; 9: 678-85$

10. Norris DP, Grimes DT. Mouse models of ciliopathies: the state of the art. Dis Model Mech 2012 ; $5: 299-312$.

11. Murdoch JN, Copp AJ. The relationship between Sonic Hedgehog signaling, cilia, and neural tube defects. Birth Defects Res Part A: Clin Mol Teratol $2010 ; 88$ : 633-52.

12. Copp AJ, Greene NDE, Murdoch JN. The genetic basis of mammalian neurulation. Nat Rev Genet $2003 ; 4: 784-93$.

13. Ribes V, Briscoe J. Establishing and interpreting graded sonic hedgehog signaling during vertebrate neural tube patterning: The role of negative feedback. Cold Spring Harb Perspect Biol $2009 ; 1:$ a002014.

14. Goetz SCS, Ocbina PJRP, Anderson KVK. The primary cilium as a Hedgehog Signal transduction machine. Methods Cell Biol 2008 ; 94 : 199-222.

15. Huangfu D, Anderson KV. Signaling from Smo to Ci/Gli: conservation and divergence of Hedgehog pathways from Drosophila to vertebrates. Development $2006 ; 133: 3-14$.

16. May SRS, Ashique AMA, Karlen MM, et al. Loss of the retrograde motor for IFT disrupts localization of Smo to cilia and prevents the expression of both activator and repressor functions of Gli. Dev Biol 2005 ; 287 : 378-89.

17. Gorivodsky M, Mukhopadhyay M, Wilsch-Braeuninger M, et al. Intraflagellar transport protein 172 is essential for primary cilia formation and plays a vital role in patterning the mammalian brain. Dev Biol $2009 ; 325: 24-32$.

18. Willaredt MA, Hasenpusch-Theil $K$, Gardner HAR, et al. A crucial role for primary cilia in cortical morphogenesis. J Neurosci $2008 ; 28: 12887-900$.

19. Stottmann RW, Tran PV, Turbe-Doan A, Beier DR. Ttc2lb is required to restrict sonic hedgehog activity in the developing mouse forebrain. Dev Biol $2009 ; 335: 166-78$.

20. Besse L, Neti M, Anselme I, et al. Primary cilia control telencephalic patterning and morphogenesis via Gli3 proteolytic processing. Development $2011 ; 138: 2079-88$

21. Wilson SL, Wilson JP, Wang C, et al. Primary cilia and Gli3 activity regulate cerebral cortical size. Dev Neurobiol $2012 ; 72$ : 1196-212

22. Magnani D, Hasenpusch-Theil K, Benadiba C, et al. Gli3 controls corpus callosum formation by positioning midline guideposts during telencephalic patterning. Cereb Cortex $2013 ; 24$ : 186-98.

23. Böse J, Grotewold L, Rüther U. Pallister-Hall syndrome phenotype in mice mutant for Gli3. Hum Mol Genet $2002 ; 11: 1129-35$.

24. Chi L, Galtseva A, Chen L, et al. Kif3a controls murine nephron number via GLI3 repressor, cell survival, and gene expression in a lineage-specific manner. PLoS One $2013 ; 8$ : e65448.

25. Tran PV, Haycraft CJ, Besschetnova TY, et al. THMI negatively modulates mouse sonic hedgehog signal transduction and affects retrograde intraflagellar transport in cilia. Nat Genet $2008 ; 40: 403-10$.

26. Goetz SC, Liem KF Jr, Anderson KV. The spinocerebellar ataxia-associated gene tau tubulin kinase 2 controls the initiation of ciliogenesis. Cell $2012 ; 151: 847-58$

27. Kim S, Tsiokas L. Cilia and cell cycle re-entry: More than a coincidence. Cell Cycle $2011 ; 10: 2683-90$.

28. Chizhikov VV, Davenport J, Zhang $Q$, et al. Cilia proteins control cerebellar morphogenesis by promoting expansion of the granule progenitor pool. J Neurosci $2007 ; 27: 9780-9$.

29. Spassky N, Han YG, Aguilar A, et al. Primary cilia are required for cerebellar development and Shhdependent expansion of progenitor pool. Dev Biol 2008 ; 317 : 246-59.

30. Aguilar A, Meunier A, Strehl L, et al. Analysis of human samples reveals impaired SHHdependent cerebellar development in Joubert syndrome/Meckel syndrome. Proc Natl Acad Sci USA 2012 ; 109 : 16951-6.

31. Paridaen JTML, Wilsch-Bräuninger M, Huttner WB. Asymmetric inheritance of centrosome-associated primary cilium membrane directs ciliogenesis after cell division. Cell $2013 ; 155$ : 333-44.
32. Anderson CT, Stearns T. Centriole age underlies asynchronous primary cilium growth in mammalian cells. Curr Biol $2009 ; 19$ : 1498-502.

33. Wang X, Tsai JW, Imai JH, et al. Asymmetric centrosome inheritance maintains neural progenitors in the neocortex. Nature 2009 ; 461 : 947-55.

34. Wilsch-Brauninger M, Peters J, Paridaen JTML, Huttner WB. Basolateral rather than apical primary cilia on neuroepithelial cells committed to delamination. Development 2011 ; 139 : 95-105.

35. Das RM, Storey KG. Apical abscission alters cell polarity and dismantles the primary cilium during neurogenesis. Science $2014 ; 343: 200-4$.

36. Lehtinen MK, Zappaterra MW, Chen X, et al. The cerebrospinal fluid provides a proliferative niche for neural progenitor cells. Neuron $2011 ; 69: 893-905$

37. Dubreuil V, Marzesco AM, Corbeil D, et al. Midbody and primary cilium of neural progenitors release extracellular membrane particles enriched in the stem cell marker prominin-1.J Cell Biol 2007 ; 176 : 483-95.

38. Higginbotham H, Guo J, Yokota $Y$, et al. Arl13b-regulated cilia activities are essential for polarized radial glial scaffold formation. Nat Neurosci 2013 ; $16: 1000-7$.

39. Kondo S, Sato-Yoshitake R, Noda Y, et al. KIF3A is a new microtubule-based anterograde motor in the nerve axon. J Cell Biol 1994 ; 125 : 1095-107.

40. Delaval B, Delaval B, Bright A, et al. The cilia protein IFT88 is required for spindle orientation in mitosis. Nature $2011 ; 13: 461-8$.

41. Higginbotham $H$, , Eom TY, Mariani LE, et al. Arll3b in primary cilia regulates the migration and placement of interneurons in the developing cerebral cortex. Dev Cell $2012 ; 23: 925-38$

42. Baudoin J-P, Viou L, Launay PS, et al. Tangentially migrating neurons assemble a primary cilium that promotes their reorientation to the cortical plate. Neuron $2012 ; 76$ : 1108-22.

43. Molyneaux BJB, Arlotta PP, Menezes JRLJ, Macklis JDJ. Neuronal subtype specification in the cerebral cortex. Nat Rev Neurosci $2007 ; 8$ : 427-37.

44. Donahoo A-LS, Richards LJ. Understanding the mechanisms of callosal development through the use of transgenic mouse models. Semin Pediat Neurol 2009 ; 16 : 127-42.

45. Benadiba C, Magnani D, Niquille M, et al. The ciliogenic transcription factor RFX3 regulates early midline distribution of guidepost neurons required for corpus callosum development. PLoS Genet 2012 ; 8 : e1002606.

46. Tadenev ALD, Kulaga HM, May-Simera HL, et al. Loss of Bardet-Biedl syndrome protein-8 (BBS8) perturbs olfactory function, protein localization, and axon targeting. Proc Natl Acad Sci USA $2011 ; 108$ : 10320-5.

47. Ferland RJR, Eyaid WW, Collura RVR, et al. Abnormal cerebellar development and axonal decussation due to mutations in AHIl in Joubert syndrome. Nat Genet $2004 ; 36: 1008-13$.

48. Poretti A, Boltshauser $\varepsilon$, Loenneker T, et al. Diffusion tensor imaging in Joubert syndrome. Am J Neuroradiol 2007 ; 28 : 1929-33.

49. Spassky NN, Merkle FTF, Flames NN, et al. Adult ependymal cells are postmitotic and are derived from radial glial cells during embryogenesis. J Neurosci $2005 ; 25: 10-8$.

50. Narita K, Kawate T, Kakinuma N, Takeda S. Multiple primary cilia modulate the fluid transcytosis in choroid plexus epithelium. Traffic $2010 ; 11: 287-301$.

51. Banizs B. Dysfunctional cilia lead to altered ependyma and choroid plexus function, and result in the formation of hydrocephalus. Development 2005 ; $132: 5329-39$.

52. Carter CS, Vogel TW, Zhang $Q$, et al. Abnormal development of NG2+PDGFR- $\alpha^{+}$ neural progenitor cells leads to neonatal hydrocephalus in a ciliopathy mouse model. Nat Med $2012 ; 18: 1797-804$

53. Lancaster MA, Gopal DJ, Kim J, et al. Defective Wnt-dependent cerebellar midline fusion in a mouse model of Joubert syndrome. Nat Med 2011 ; 17 : 726-31.

54. Kumamoto N, Gu Y, Wang J, et al. A role for primary cilia in glutamatergic synaptic integration of adult-born neurons. Nat Neurosci 2012 ; 15 : 399405-S1.

55. Guadiana SM, Semple-Rowland S, Daroszewski D, et al. Arborization of dendrites by developing neocortical neurons is dependent on primary cilia and type 3 adenylyl cyclase. J Neurosci 2013 ; 33 : 2626-38.

56. Han Y-G, Spassky N, Romaguera-Ros M, et al. Hedgehog signaling and primary cilia are required for the formation of adult neural stem cells. Nat Neurosci $2008 ; 11: 277-84$.

57. Breunig JJJ, Sarkisian MRM, Arellano JIJ, et al. Primary cilia regulate hippocampal neurogenesis by mediating sonic hedgehog signaling. Proc Natl Acad Sci USA 2008 ; 105 : 13127-32.

58. Bishop GA, Berbari NF, Lewis J, Mykytyn K. Type III adenylyl cyclase localizes to primary cilia throughout the adult mouse brain. J Comp Neurol 2007 ; $505: 562-71$. 


\section{RéFÉRENCES}

59. Green JA, Gu C, Mykytyn K. Heteromerization of ciliary G protein-coupled receptors in the mouse brain. PLoS One $2012 ; 7$ : e46304.

60. Davenport JR, Watts AJ, Roper VC, et al. Disruption of intraflagellar transport in adult mice leads to obesity and slow-onset cystic kidney disease. Curr Biol 2007 ; 17 : 1586-94.

61. Loktev AV, Zhang $\mathrm{Q}$, Beck JS, et al. A BBSome subunit links ciliogenesis, microtubule stability, and acetylation. Dev Cell 2008; 15:854-65.

62. Sang L, Miller JJ, Corbit KC, et al. Mapping the NPHP-JBTS-MKS protein network reveals ciliopathy disease genes and pathways. Cell $2011 ; 145: 513-28$.

63. Kriegstein A, Alvarez-Buylla A. The glial nature of embryonic and adult neural stem cells. Annu Rev Neurosci 2009; 32 : 149-84

64. Marín O, Valiente M, Ge X, Tsai LH. Guiding neuronal cell migrations. Cold Spring Harb Perspect Biol $2010 ; 2$ : a001834

65. Fort C, Bastin P. Élongation de l'axonème et dynamique du transport intraflagellaire. Med Sci (Paris) $2014 ; 30: 955-61$

66. Benmerah A. La poche ciliaire : fruit des liaisons du centrosome avec le trafic vésiculaire. Med Sci (Paris) $2014 ; 30: 962-7$.

67. Bachmann-Gagescu R. Complexité génétique des ciliopathies et identification de nouveaux gènes. Med Sci (Paris) 2014 ; $30: 1011-23$

68. Métin C. Cils et migration neuronale. Med Sci (Paris) 2014 ; $30: 991-5$.
69. Delgehyr N, Spassky N. Cil primaire, cycle cellulaire et prolifération. Med Sci (Paris) $2014 ; 30: 976-9$.

70. Taulet N, Delaval B. De nouvelles fonctions extraciliaires pour les protéines ciliaires. Quelles conséquences sur l'apparition de ciliopathies? Med Sci (Paris) $2014 ; 30: 1040-50$.

71. Chennen K, Scerbo MJ, Dollfus H, et al. BBS : cils et obésité ; de la génétique à l'approche intégrative. Med Sci (Paris) 2014 ; 30 : 1034-9.

\section{REMERCIEMENTS}

Je remercie les membres du laboratoire pour leur relecture attentive du manuscrit et les critiques constructives qu'ils m'ont faites: Sylvie Schneider-Maunoury, Christine Vesques, Guillaume Pezeron et bien sûr Martin Catala pour la partie médicale. Merci aussi à Christine Métin pour ses commentaires avisés.

TIRÉS À PART

C. Laclef

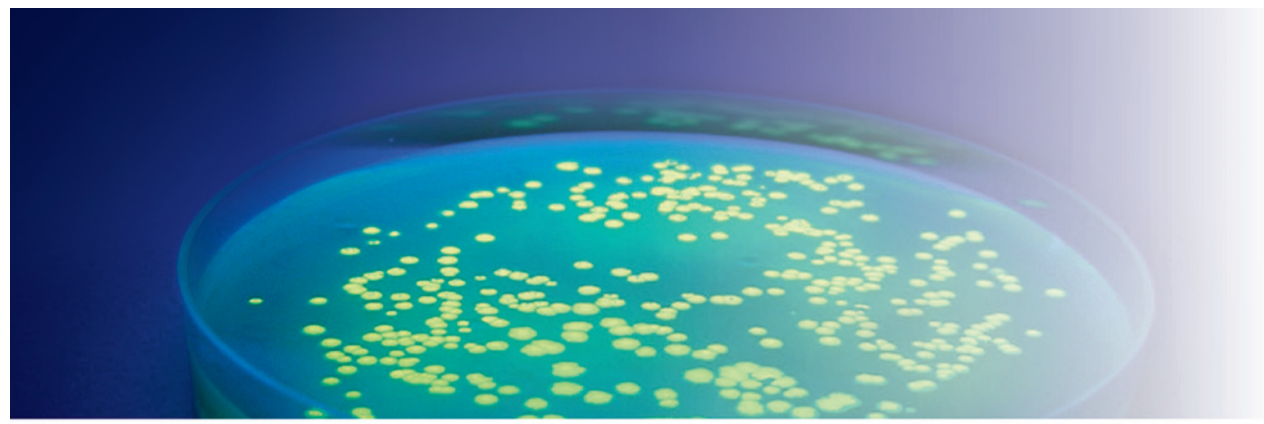

\section{PlasmidFactory}

\section{Service ADN minicirculaire et ADN plasmidique}

- Production d'ADN minicirculaire et de plasmides en fonction des besoins du client

- Service en stock de gènes rapporteurs, plasmides ou ADN minicirculaire (GFP, lacZ, luc, miniS/MAR etc.)

O Service en stock pour les plasmides auxiliaires AAV et d'emballage (par ex. pDG/pDP)

O Qualité certifiée et constante

○ Option High Quality: ADN pour production GMP d'ARN et de vecteurs viraux

O QC comprenant une analyse CGE des topologies de l'ADN Études sur la stabilité et la conservation 